\title{
An Intelligent Algorithm of Bi-level Programming Model for Three-Stage Supply Chain
}

\author{
Rui Wang, Shuang Zhao, Renqiao Rao \\ School of Business, Sichuan University, Chengdu, 610064, P. R. China
}

\begin{abstract}
In this paper, we established the optimal strategy model and compiled the hybrid intelligent algorithm to solve this model, using the fuzzy random simulation and enumeration method to find out manufacturer's optimal order quantity, optimal production and optimal R\&D investment; supplier's optimal production, optimal participation rates as well as the corresponding profits; retailer's optimal order quantity and the corresponding profit. We get conclusions that when manufacturer and supplier collaboratively research and develop as well as give quantity discounts for retailer, the production volume of supplier and manufacturer has basically reached the upper limit.
\end{abstract}

Keywords-supply chain; programming; hybrid intelligent algorithm.

\section{INTRODUCTION}

A supply chain usually includes raw material suppliers, manufacturers, distributors, wholesalers, and retailers. Each member in the supply chain tries to gain advantages whether in a competitive or in a cooperative relationship [1]. it is feasible to create a mechanism among the members for coordinating resources and individuals, which can increase the profit of the entire supply chain. This is collaboration which we usually referred.

The significance of uncertainty has prompted a number of researchers to address stochastic parameters in tactical level supply chain planning involving distribution of raw materials and products[2][3][4]. At the strategic level, there is a great deal of research in the facility location component of supply chain network design under uncertainty [5]. Moinzadeh and Lee [6], Yao [7] studied the inventory model with uncertain yield and came up with that the optimal strategies of two types of models are formally similar as they are separately provided with determine yield and uncertain yield while both are essentially difference.

\section{PROBLEM DESCRIPTION}

This paper considers a three-stage supply chain consisted of a supplier, a manufacturer and a retailer, in which the supplier, manufacturer and retailer are not in the same country. In the first phase, the manufacturer needs to combine the information of market conditions with its own factors to determine the order quantity of this phase provided to the supplier $q_{m s}$ and a price discount program for the retailers $p_{m r}(\theta)$; In the second phase, the supplier combines the information given by the manufacturer with its own situation to determine its tasks proportion of $R \& D$ joined with the manufacturer $w$, that is, in the R\&D project, workload borne by the supplier is $a \times w$ and the retailer determines the order quantity $q_{r m}$ according to the principle of maximizing benefits; in the third phase, according to the supplier's feedback information, the manufacturer determines the amount of work committed to the design of new products $a$, then determines the value of the production volume $q_{m}$ according to retailer's feedback information, and adjust the parameters $\theta$ of $p_{m r}(\theta)$, latterly, the three repeatedly adjusted until it reaches equilibrium.

\section{MODELLING}

\section{A. The basic model.}

In this section, we first discuss the coordination among the supplier, the manufacturer and the retailer in the situation that the manufacturer carries out research works alone and doesn't give the retailer at a discount.

Supplier profit:

$$
\Pi_{Z}^{S}\left(q_{s}\right)=\left[\left(p_{s m}-t c_{s m}\right) \times q_{m s}-p c_{s} \times q_{s}-\left(q_{m s}-q_{s}\right)^{+} \times p c_{e}\right] / r_{s}
$$

Manufacturer profit:

$$
\begin{aligned}
& \Pi_{Z}^{M}\left(a, q_{m}, q_{m s}\right)=\left[\left(p_{m r}-t c_{m r}\right) \times q_{r m}-p c_{m} \times q_{m}-\left(q_{r m}-q_{m}\right)^{+} \times p c_{e}^{\prime}\right] / r_{m} \\
& -\left[p_{s m} \times\left(1+t_{m}\right)+t c_{s m} \times t_{m}\right] \times q_{m s} / r_{s}-a \\
& \text { Retailer profit: } \\
& \quad \Pi_{Z}^{R}\left(q_{r m}, d\right)=p_{r} s_{r} / r_{r}-\left[p_{m r} \times\left(1+t_{r}\right)+t c_{m r} \times t_{r}\right] \times q_{r m} / r_{m}
\end{aligned}
$$

According to market operation mechanism, we can be established as follows bi-level programming:

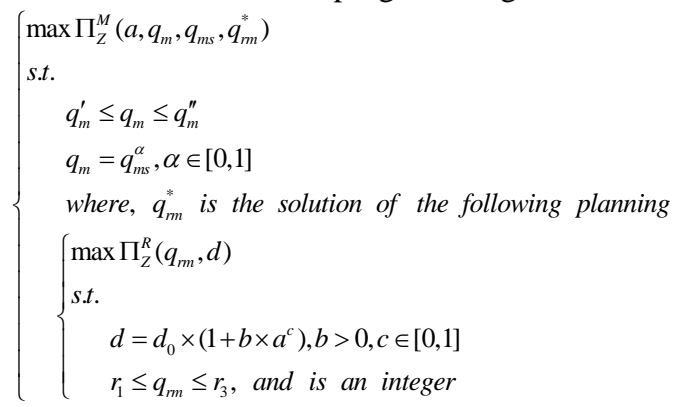

\section{B. Dual mechanism model.}

This section mainly discusses the coordination among the supplier, the manufacturer and the retailer in the situation that the manufacturer combining the supplier 
carries out research works and the manufacturer gives the retailer at a discount.

Supplier profit:

$$
\left.\Pi_{z}^{\prime s}\left(w, q_{s}\right)=\left(p_{s m}-t c_{s m}\right) \times q_{n s}-p c_{s} \times q_{s}-\left(q_{n s}-q_{s}\right)^{+} \times p c_{e}\right) / r_{s}-a \times w
$$

Manufacturer profit:

$$
\begin{aligned}
& \Pi_{Z}^{\prime M}\left(p_{m r}(\theta), q_{m}, q_{m s}, a\right)=\left[\left(p_{m r}(\theta)-t c_{m r}\right) \times q_{r m}-p c_{m} \times q_{m}-\left(q_{r m}-q_{m}\right)^{+} \times p c_{e}^{\prime}\right] / r_{m} \\
& -\left[p_{s m} \times\left(1+t_{m}\right)-t c_{s m} \times t_{m}\right] \times q_{m s} / r_{s}-(1-w) a
\end{aligned}
$$

Retailer profit:

$$
\Pi_{Z}^{\prime R}\left(q_{r m}, d\right)=p_{r} s_{r} / r_{r}-\left[p_{m r}(\theta) \times\left(1+t_{r}\right)+t c_{m r} \times t_{r}\right] \times q_{r m} / r_{m} \quad(7)
$$

According to market operation mechanism, we can establish a bilayer multi-objective planning, as follows:

$$
\left\{\begin{array}{l}
\max \Pi_{z}^{\prime M}\left(a, p_{m r}(\theta), q_{m}, q_{m s} w^{*}, q_{r m}^{*}\right) \\
\quad q_{m}^{\prime} \leq q_{m} \leq q_{m}^{\prime \prime} \\
d=d_{0} \times\left(1+b \times a^{c}\right), b>0, c \in[0,1] \\
q_{m}=q_{m s}^{\alpha}, \alpha \in[0,1] \\
b=f(w) \\
0<\theta<1 \\
t c_{m r}+p c_{m}<p_{m r}<p_{r}-t c_{m r} \\
\begin{array}{l}
w h e r e, w^{*}, q_{r m}^{*} \text { is the solution of the planning } \\
\max \Pi_{z}^{\prime R}\left(q_{r m}, d\right) \\
\max \Pi_{z}^{\prime s}\left(w, q_{s}\right) \\
\text { s.t. } \\
\quad q_{r m}>0, \text { and is an integer } \\
\quad \begin{array}{l}
w \in[0,1] \\
q_{s}^{\prime} \leq q_{s} \leq q_{s}^{\prime \prime}
\end{array}
\end{array}
\end{array}\right.
$$

\section{Algorithm Design}

\section{A. Fuzzy random simulation algorithm to solute retailers} ordering strategy.

To solve the model (4) and get the optimal ordering strategy of retailers, this paper uses fuzzy random techniques. Specific steps of the algorithm are:

Step1: According to $\xi$ obeying the probability distribution $p\left(\xi=\omega_{i}\right)=p_{i}$, we take $\omega_{m}$ randomly from sample space, and get the sample value $u=d\left(\omega_{m}\right)$ of the fuzzy random variable $d$, and then produce sample values in the level of $d\left(\omega_{m}\right)$ evenly.

Step2: The sample values of other variables are obtained by using random simulation, fuzzy simulation, and fuzzy random simulation techniques.

Step3: Traverse the feasible region of $q_{m s}$ according to the value range of the retailer's order.

Step4: Calculate the optimal value of fuzzy random variables $\Pi^{R}\left(q_{m r}\right)$.
B. The hybrid intelligent algorithm to solve the threestage supply chain model having research but no synergy.

To solve the model (4) and obtain the optimal production $q_{s}$ of supplier, optimal production $q_{m}$ and optimal R\&D investment $a$, as well as optimal order amount $q_{r m}$ of the retailer under the situations of the manufacturer developing separately and the manufacturer not giving retailer price discounts, we give the corresponding intelligent algorithm. The specific steps are as follows:

Step1: Initialize the manufacturer's decision variables $q_{m}^{0}$ and $a^{*}$, that is, Nind viable populations $\left(q_{m i}, a_{i}\right)$ of lower layer are produced in the interval $\left(q_{m}^{\prime}, q_{m}^{\prime \prime}\right)$ and $\left(0, a^{\prime \prime}\right)$, where $i=1,2, \ldots$, Nind .

Step2: The specific values are produced for each unknown variables by fuzzy simulation, random simulation, and fuzzy random simulation technology.

Step3: Substitute each variable into the lower layer, and obtain optimal value using the method in section 4.1, and then return to the upper planning.

Step4: Each chromosome $\left(q_{m i}, a_{i}\right)$ is substituted into the manufacturer's profit function $\Pi_{B}^{\prime M}\left(p_{m r}, q_{m}, q_{m s}, q_{r m}^{*}\right)$ to determine the fitness of chromosome, where $i=1,2, \ldots$, Nind .

Step5: The fitness of the chromosomes array in descending order, and choose them by roulette method.

Step6: New chromosomes are done the crossover and mutation, which generate new chromosomes $\left(q_{m i}^{\text {new }}, a_{i}^{\text {new }}\right)$ satisfying the constraints.

Step7: New chromosomes are substituted into the lower layer, and obtain the optimal value by using the method in section 4.1, and return to the upper layer.

Step8: We evaluate new chromosomes by the objective function $\Pi_{Z}^{M}$ of the manufacturer, and the individual for best fitness is $\left(q_{m i}^{*}, a^{*}\right),(i=1,2, \cdots$, Nind $)$.

Step9: Test the termination conditions of upper layer. If it reaches genetic algebra, then terminate. Unless the termination condition is satisfied, then we transfer to step 4.

C. Hybrid intelligent algorithm for solving the three-stage supply chain model of coordinating mechanism.

To solve the model (8) and obtain the optimal production $q_{s}^{*}$, the optimal ratio of participation in $R \& D$ $w^{*}$ of supplier, optimal price $p_{m r}{ }^{*}(\theta)$, optimal production $q_{m}$ and optimal R\&D investment $a^{*}$ of manufacturer, as well as optimal order amount $q_{r m}^{*}$ of the retailer under the situations that the supplier participates in research with manufacturers and the manufacturer gives retailer price discounts, we give the corresponding intelligent algorithm:

Step1: Initialize the manufacturer's decision variables $\theta^{0}, q_{m}^{0}$ and $a^{*}$, that is, Nind viable populations 
$\left(\theta_{i}, q_{m i}, a_{i}\right)$ of lower layer are produced in the interval $[0,1),\left(q_{m}^{\prime}, q_{m}^{\prime \prime}\right)$ and $\left(0, a^{\prime \prime}\right)$, where $i=1,2, \ldots$, Nind .

Step2: The specific values are produced for each unknown variables by fuzzy simulation, random simulation, and fuzzy random simulation technology.

Step3: Substitute each variable into the lower layer, and obtain optimal value using the method in section 4.2, and then return to the upper planning.

Step4: Each chromosome $\left(\theta_{i}, q_{m i}, a_{i}\right)$ is substituted into the manufacturer's profit function to determine the fitness of chromosome, where $i=1,2, \ldots$, Nind .

Step5: The fitness of the chromosomes array in descending order, and choose them by roulette method.

Step6: New chromosomes are done the crossover and mutation, which generate new chromosomes $\left(\theta_{i}^{\text {new }}, q_{m i}^{\text {new }}, a_{i}^{\text {new }}\right)$ satisfying the constraints.

Step7: New chromosomes are substituted into the lower layer, and obtain the optimal value by using the method in section 4.2, and return to the upper layer.

Step8: We evaluate new chromosomes by the objective function of the manufacturer, and the individual for best fitness is $\left(\theta_{i}^{*}, q_{m i}^{*}, a^{*}\right),(i=1,2, \cdots$, Nind $)$.

Step9: Test the termination conditions of upper layer. If it reaches genetic algebra, then terminate. Unless the termination condition is satisfied, then we transfer to step 4.

\section{V.NUMERICAL EXAMPLES}

In order to verify the feasibility of the above models and algorithms, the basic parameters of the model are as follows:

Function: In the example, we assume that the deeper supplier involves in research, the higher the overall $R \& D$ efficiency of the supply chain is. But if the supplier involvement in research is beyond the manufacturer, $R \& D$ efficiency of the supply chain will decrease. That is: $b=f\left(w^{2}\right)=a_{1} w^{2}+a_{2} w+a_{3}$. Where, $a_{1}<0$.

Variable value: The market demand $d$ is a fuzzy random variable, and its probability distribution and fuzzy values are shown in Table 1 , which $\mu$ is a triangular fuzzy number and random variable $\omega_{i} \in$ [weak market, the market in general, strong market]. Other variable values are shown in Table II, Table III, and Table IV.

TABLE I. FUZZY RANDOM DEMAND

\begin{tabular}{llll}
\hline $\boldsymbol{p}\left(\xi=\omega_{i}\right)$ & 0.2 & 0.6 & 0.2 \\
\hline$d\left(w_{i}\right)$ & $u_{1}\left(\omega_{1}\right)=(680,750,820)$ & $u_{2}\left(\omega_{2}\right)=(800,900,1000)$ & $u_{3}\left(\omega_{3}\right)=(950,1010,1070)$ \\
\hline \multicolumn{4}{c}{ TABLE II. EXCHANGE RATE } \\
\hline $\boldsymbol{r}_{\boldsymbol{s}}$ & $\boldsymbol{r}_{\boldsymbol{m}}$ & $\boldsymbol{r}_{\boldsymbol{r}}$ \\
\hline$N(6,4)$ & $N(2,0.25)$ & $N(1,0.25)$ \\
\hline
\end{tabular}

TABLE III. RELEVANT COST PARAMETERS

\begin{tabular}{lcccccc}
\hline Variable & $\boldsymbol{p}_{\boldsymbol{s} \boldsymbol{m}}$ & $\boldsymbol{t} \boldsymbol{c}_{\boldsymbol{s m}}$ & $\boldsymbol{p \boldsymbol { c } _ { s }}$ & $\boldsymbol{p \boldsymbol { c } _ { \boldsymbol { e } }}$ & $\boldsymbol{p \boldsymbol { c } _ { \boldsymbol { m } }}$ & $\boldsymbol{p}_{\boldsymbol{m}}$ \\
\hline Value & 20 & 2 & 2 & 3.5 & 25 & 80 \\
\hline
\end{tabular}

TABLE IV. OTHER PARAMETERS

\begin{tabular}{lcccccccc}
\hline Variable & $q_{m}^{\prime}$ & $q_{m}^{\prime \prime}$ & $\alpha$ & $q_{s}^{\prime \prime}$ & $t_{m}$ & $a_{1}$ & $a_{2}$ & $a_{3}$ \\
\hline Value & 350 & 1000 & 0.95 & 800 & 0.1 & -1 & 1.2 & $1 / 30$ \\
\hline
\end{tabular}

By genetic algorithm, combined with random simulation and fuzzy random simulation technology using MATAB (R2011a), we obtain an optimization result after 250 generations within the feasible region:

TABLE V. THE OPTIMAL STRATEGY AND PROFITS OF SUPPLIER, MANUFACTURER AND RETAILER

\begin{tabular}{c|c|c|c|c|c|c|c|c|c|c|c|c|c}
\hline Variable & $\theta$ & $\boldsymbol{p}_{\boldsymbol{m}}$ & $\boldsymbol{q}_{\boldsymbol{m}}$ & $\boldsymbol{q}_{\boldsymbol{m}}$ & $\boldsymbol{a}$ & $\boldsymbol{q}_{\boldsymbol{r m}}$ & $\boldsymbol{q}_{\boldsymbol{s}}$ & $\boldsymbol{w}$ & $\Pi_{M}$ & $\Pi_{S}$ & $\Pi_{R}$ & $\Pi_{S R}$ \\
\hline 1 & $/$ & 80 & 720 & 1018 & 365 & 720 & 800 & $/$ & 19309 & 4397 & 20389 & 12393 \\
2 & 0.0375 & 77 & 988 & 1420 & 320 & 988 & 800 & 0.45 & 25321 & 6003 & 29866 & 17934.5 \\
\hline
\end{tabular}

We can see that the optimal profit of supplier, manufacturer and retailer has rose by $31.14 \%, 36.52 \%$ and $31.73 \%$ respectively compared to the case of manufacturers doing R\&D alone and no quantity discounts. Moreover, both production volume of the supplier and the manufacturer have basically reached the limit, indicating that the production resources have used reasonably

\section{CONCLUSIONS}

This paper studies the international supply chain in the situations that the supplier participates the $R \& D$ with manufacturer and manufacturer offers the retailer price discounts. The optimal policy of the supplier, the manufacturer and the retailer obtained in both cases by building random fuzzy double multi-objective model and using fuzzy random simulation technology combined with genetic algorithm.

\section{REFERENCES}

[1] C. F. Hsueh, and M. S. Chang, "Equilibrium analysis and corporate social responsibility for supply chain integration,” European Journal of Operational Research, vol. 190, pp. 116-129, 2008.

[2] R. K. M. Cheung, and W. B. Powell, "Models and algorithms for distribution problems with uncertain demands,". Transportation Science, vol. 30, pp. 43-59, 1996.

[3] H. Van Landeghem, and H. Vanmaele, "Robust planning: a new paradigm for demand chain planning," Journal of Operations Management, vol. 20, pp. 769-783, 2002.

[4] C. S. Yu, and H. L. Li, "A robust optimization model for stochastic logistic problems," International Journal of Production Economics, vol. 64, pp. 385-397, 2000.

[5] S. H. Owen and M. S. Daskin, "Strategic facility location: A review," European Journal of Operational Research, vol. 111, pp. 423-447, 1998. 
[6] K. Moinzadeh and H. L. Lee, "A continuous-review inventory model with constant resupply time and defective items," Naval research logistics quarterly, vol. 34. Pp. 457-467, 1987.

[7] D. D. Yao. "Optimal run quantities for an assembly system with random yields,” IIE Transactions, vol. 20, pp. 399-403, 1988.
[8] M. Fisher, and A. Raman, "Reducing the cost of demand uncertainty through accurate response to early sales," Operational Research, vol. 44, pp. 87-99, 1996. 